\title{
Breast Cancer Care in India: The Current Scenario and the Challenges for the Future
}

\author{
Gaurav Agarwal Pooja Ramakant \\ Department of Endocrine and Breast Surgery, Sanjay Gandhi Postgraduate Institute of Medical Sciences, Lucknow, India
}

\section{Key Words}

Breast cancer: early detection, epidemiology, guidelines, incidence - Locally advanced disease . Metastatic disease . Multimodal treatment . Mastectomy

\section{Summary}

The incidence of breast cancer is low in India, but rising. Breast cancer is the commonest cancer of urban Indian women and the second commonest in the rural women. Owing to the lack of awareness of this disease and in absence of a breast cancer screening program, the majority of breast cancers are diagnosed at a relatively advanced stage. The quality of care available for breast cancer patients varies widely according to where the patient is treated. Although there are some centers of excellence providing multimodality protocol-based treatment at par with the best anywhere in the world, the vast majority of breast cancer patients undergo inadequate and inappropriate treatment due to lack of high-quality infrastructure and sometimes skills, and above all financial resources. The recent emphasis on health education, early diagnosis of cancers, and more public facilities for cancer treatment are expected to bring about the much needed improvement in breast cancer care in India.

\author{
Schlüsselwörter \\ Brustkrebs: Früherkennung, Epidemiologie, \\ Leitlinien, Inzidenz · Lokal fortgeschrittene Erkrankung · \\ Metastasierte Erkrankung - Multimodale Behandlung · \\ Mastektomie
}

\section{Zusammenfassung}

Die Brustkrebsinzidenz in Indien ist niedrig aber steigend. Brustkrebs ist die häufigste Krebserkrankung unter Stadtbewohnerinnen und die zweithäufigste in ländlichen Gebieten. Auf Grund der mangelnden Aufklärung bezüglich der Erkrankung und da kein Brustkrebs-Screeningprogramm existiert, werden die meisten Brustkrebsfälle in einem relativ späten Stadium diagnostiziert. Die Qualität der medizinischen Versorgung von Brustkrebspatientinnen variiert sehr stark und ist abhängig davon, wo die Behandlung stattfindet. Obwohl eine geringe Anzahl an leistungsfähigen Zentren zur Verfügung steht, in denen multimodale, auf Protokollen basierende Behandlung angeboten wird, die mit den besten Zentren anderswo in der Welt vergleichbar sind, wird die Mehrheit der Brustkrebspatientinnen unzureichend und unangemessen behandelt. Ursache dafür sind der Mangel an einer hochwertigen Infrastruktur und manchmal auch an Fähigkeiten sowie vor allem an finanziellen Mitteln. In letzter Zeit wird der medizinischen Aufklärung mehr Aufmerksamkeit geschenkt, ebenso der Krebsfrühdiagnose sowie dem Ausbau öffentlicher Krebsbehandlungszentren, dies wird hoffentlich die dringend benötigte Verbesserung in der Betreuung von Brustkrebspatientinnen in Indien mit sich bringen.

\begin{tabular}{ll}
\hline KARGER & $\oplus$ 2008 S. Karger GmbH, Freiburg \\
Fax +49 761 452 07 14 & Accessible online at: \\
$\begin{array}{l}\text { E-mail Information@Karger.de } \\
\text { www.karger.com }\end{array}$ & www.karger.com/brc
\end{tabular}




\section{Introduction}

India is a sub-continent with wide ethnic, cultural, religious, and economic diversity and variation in the health care infrastructure. The health care facility pattern is heterogeneous, with numerous regions where the benefits of the awareness, early diagnosis, and multidisciplinary treatment programs have not reached. With rising incidence and awareness, breast cancer is the commonest cancer in urban Indian females, and the second commonest in the rural Indian women [1]. The numerous myths and ignorance that prevail in the Indian society result in an unrealistic fear of the disease [2]. Breast cancer awareness programs are more concentrated in the cities and have not reached the remote and rural parts of the country [2, $3]$. Women often do not present for medical care early enough due to various reasons such as illiteracy, lack of awareness, and financial constrains. It is hardly surprising that the majority of breast cancer patients in India are still treated at locally advanced and metastatic stages [2, 4]. Lack of an organized breast cancer screening program, paucity of diagnostic aids, and general indifference towards the health of females in the predominantly patriarchal Indian society do not help early diagnosis of breast cancer. A multidisciplinary approach to breast cancer treatment that is so vital is available only at a few select regional centers. This review aims at providing an overview of the available data and views on breast cancer care in India. Data from the various cancer registries, publications/presentations from individual institutions, and the data from SGPGIMS Lucknow - a major tertiary care teaching hospital of North India - have been used. The data available on various issues relating breast cancer care in India is scant and heterogenous. There is no central cancer registry to provide comprehensive nationwide data. The only credible data on a large proportion of population is available from the population-based cancer registries (PBCRs) - both urban and rural - and the various hospital-based cancer registries which work under the national cancer registries program of the Indian Council of Medical Research (ICMR). There are numerous other non-ICMR cancer registries organized and run by hospitals and institutions. One major hindrance in collation of data from these diverse registries is the lack of uniform methods of data collection and storage. This makes any meaningful interpretation of nationwide data an arduous task, and any efforts at this are often viewed with suspicion.

\section{Epidemiology and Demography of Breast Cancer in India}

Breast cancer is the commonest cancer in women worldwide with a widely variable incidence between countries and regions. The developed countries with a small proportion of the world population account for almost $50 \%$ of breast cancers diagnosed worldwide [5]. The lowest breast cancer incidence is reported from Far Eastern and South-East Asian countries $[1,2]$. In the developing countries of Asia, the health care burden on account of breast cancer has been steadily mounting. It is expected that in the coming decades, these countries would account for majority of new breast cancer patients diagnosed globally. Over 100,000 new breast cancer patients are estimated to be diagnosed annually in India $[2,6]$. As per the ICMR-PBCR data, breast cancer is the commonest cancer among women in urban registries of Delhi, Mumbai, Ahmedabad, Calcutta, and Trivandrum where it constitutes $>30 \%$ of all cancers in females [7]. In the rural PBCR of Barshi, breast cancer is the second commonest cancer in women after cancer of the uterine cervix [7]. The age standardized incidence rates (AARs) range from 6.2 to 39.5 per 100,000 Indian women. The AARs vary from region, ethnicity, religion, with the highest incidence reported at 48.3 per 100,000 women in the Parsi community of Mumbai [7]. The incidence of this disease has been consistently increasing, and it is estimated it has risen by $50 \%$ between 1965 and 1985 [8]. The rise in incidence of $0.5-2 \%$ per annum has been seen across all regions of India and in all age groups but more so in the younger age groups ( $<45$ years) [9]. In general, breast cancer has been reported to occur a decade earlier in Indian patients compared to their western counterparts. While the majority of breast cancer patients in western countries are postmenopausal and in their 60ies and 70ies, the picture is quite different in India with premenopausal patients constituting about $50 \%$ of all patients (SGPGIMS Lucknow data) [2]. More than $80 \%$ of Indian patients are younger than 60 years of age. The average age of patients in 6 hospital-based cancer registries ranged from 44.2 years in Dibrugarh, 46.8 years in Delhi, 47 years in Jaipur, to 49.6 years in Bangalore and Chennai. The average age of breast cancer patients has been reported to be 50-53 years in various population-based studies done in different parts of the country [7]. A significant proportion of Indian breast cancer patients are younger than 35 years of age. This proportion varies between $11 \%$ (Tata Memorial Hospital (TMH) Mumbai) [10] to 26\% (SGPGIMS Lucknow) [2]. Young age has been associated with larger tumor size, higher number of metastatic lymph nodes, poorer tumor grade, low rates of hormone receptor-positive status, earlier and more frequent locoregional recurrences, and poorer overall survival $[11,12]$.

\section{Reproductive History and Breast Cancer in Indians}

Marriage at an early age, early and multiple childbirths, and breastfeeding of all children for a long period of time is the norm in most Indian societies. However, the urban educated class is moving away from this trend, with late-age childbirth and little or no breastfeeding due to changing social values and the demands of jobs on working women. These changes may be partly responsible for the increasing trend of breast 
cancer incidence. Nulliparity and late age at first childbirth are consistently observed reproductive risk factors. A case control study in Mumbai indicated that compared to married women, single women had a 4-5-fold higher risk for developing breast cancer in the age group of 40-54 and above [13]. In another study, nulliparous women had a 2.2-fold higher risk than parous women [14].

\section{Familial and Genetic Breast Cancer in Indian Women}

Almost a third of all breast cancer patients are believed to have familial disease pattern, and some $5 \%$ are believed to be hereditary, with the BRCA1 and BRCA2 gene mutations having been identified as the major genetic causes. In an Indian study on 226 breast cancer patients, $20.7 \%$ had a positive family history [15]. On the contrary, numerous other studies have reported a low rate of familial pattern of breast cancer in Indian patients. This is particularly interesting given the relatively young age of Indian breast cancer patients. At SGPGIMS Lucknow, only about $5 \%$ of all patients managed had a definite family history of breast and/or ovarian cancer in first degree relatives, similar to the figures available from other Indian centers. Genetic screening/diagnosis is not routinely performed in most Indian center due to paucity of funds and facilities. As a result, there is scarce data on the genetic composition and BRCA1/2 mutations in Indian patients. The available studies hint at a rather low incidence of BRCA mutations. In most populations, $6-10 \%$ of patients with breast cancer have mutation in BRCA gene irrespective of their family history. Though there are no robust figures, various Indian studies have reported BRCA mutations in 9-25\% of familial breast cancer cases [16-20]. Hedau et al. [19] demonstrated 3 novel BRCA1 mutations including a founder Ashkenazi Jewish BRCA1 mutation in Indian breast cancer patients [19].

\section{Association of Religion with Breast Cancer}

Indian society is a secular mix of numerous races and religions, which results in substantial variation in lifestyle patters and customs followed by individuals of different religious faiths. In Mumbai, breast cancer incidence rates are highest among Parsis and Christians and lowest among Jains and Buddhists. The possible reasons for high breast cancer incidence in the Parsi community are their westernized lifestyle, consanguineous marriages, and late age of marriage and childbirth [21]. In another study in Chennai, rates were highest in Christians followed by Hindus and Muslims [21]. Similar results have been reported from a study performed in the South Indian city of Thiruvananthapuram [22]. Religion seems to not have any bearing on any other clinical and pathological variables of breast cancer patients [21].

\section{Detection of Breast Cancer in India}

Almost all Indian breast cancer patients self-detect their disease at a stage when it presents with a palpable lump, or even at a stage when it has resulted in secondary changes such as local skin or chest wall changes or distant metastases [2]. Manifestations of invasion of the skin, such as skin edema, ulceration, and fungation, and/or of the chest wall are evident in almost half of all Indian patients who are free of any distant metastases. This is similar to the picture in many other countries with limited resources, and similar reports are available from the developing Arab World, natives of Mexico, and the Indian subcontinent [23]. Metastatic disease outside of the ipsilateral axilla is evident in $6-25 \%$ of Indian patients [22]. A combination of many factors seems responsible for this late stage of detection. These include lack of awareness about the disease resulting in few women following self breast examination or opting for a periodic examination by a healthcare worker or mammography for breast cancer screening. Even after detecting an abnormality such as a lump, the visit to a doctor for diagnosis and treatment is postponed substantially, as the initial manifestations - lumps, etc. - are not associated with pain or other troublesome symptoms. Inadequate diagnostic facilities at the peripheral/ community health centers close to a woman's home act as a deterrent from seeking specialist advice. That may mean traveling long distances, and substantial expenditure on transportation and professional fees. Lack of governmental or nongovernmental support for screening, diagnosis, and treatment costs is another major deterrent in getting timely advice and treatment. A cohort study in Chennai that included patients managed during the period of 1960-1989 reported that the age-adjusted incidence of contralateral breast cancer in patients treated for unilateral breast cancer was 7 times the incidence of unilateral breast cancer in the general population. Among women with unilateral breast cancer, the relative risk was 4.5 comparing women with and without a history of breast cancer in their mother, and 2.8 comparing women aged 21-25 years at first childbirth to those younger. Unilateral breast cancer patients not receiving any hormone treatment had a relative risk of 0.3 as compared to those receiving hormone therapy [24].

\section{TNM Staging}

Table 1 provides a summary of TNM stage at presentation at 4 major cancer centers in India. Almost $50 \%$ of patients present with locally advanced disease. The majority of patients present with stage III-b (35\% at SGPGIMS Lucknow) and III-a (27\%) [15]. Quite a few patients have large operable breast cancers and stage II-b (16\%) [15]. Some $8-10 \%$ of patients have TNM stage IV disease at presentation, and only very few (approximately 5\%) have stage I disease. In a large cohort of 
Table 1. Stage of breast cancer at presentation at 4 major cancer centers

\begin{tabular}{lcccc}
\hline \multirow{2}{*}{ Stage } & Patients, \% & & & \\
\cline { 2 - 5 } & Mumbai [3] & Trivandrum [3] & Chennai [3] & Lucknow \\
\hline I & 7.8 & 4.4 & 1 & 4 \\
II & 57.4 & 42.3 & 23 & 33 \\
III & 28.9 & 40.5 & 52 & 47 \\
IV & 5.9 & 12.8 & 24 & 9 \\
Un-staged & & & & 7 \\
\hline
\end{tabular}

patients managed over a long time period at a New Delhi hospital, stage III-b (35\%) followed by stage III-a (27\%) and II-b (16\%) predominated. Stage IV patients constituted $7.9 \%$, and only $1.4 \%$ had stage I disease [15]. At SGPGIMS Lucknow, axillary nodal metastasis is present in $>50 \%$ of patients with operable breast cancers (T1 and $\mathrm{T} 2$ disease). Axillary nodal metastases are present in almost $2 / 3$ rd of $\mathrm{T} 3$ and $\mathrm{T} 4$ patients. In a study from a large hospital in New Delhi, $80 \%$ of the patients treated for breast cancer over a period of 1 decade had metastatic disease in the axillary lymph nodes [15]. 6-25\% of Indian breast cancer patients have distant metastatic disease at presentation, with a higher incidence of skeletal metastases $[2,22]$. The metabolic consequences of skeletal metastatic disease are compounded due to a poor vitamin D and calcium intake in Indian women [2].

\section{Pathology of Breast Cancer in India}

In a study from New Delhi [15], the histo-morphological types seen in breast cancer patients indicates that invasive ductal carcinoma not otherwise specified (IDC NOS) was found to be the most common type ( $88 \%$ ) followed by infiltrating lobular carcinoma $(3.7 \%)$, colloid carcinoma $(1.1 \%)$, ductal carcinoma in situ (DCIS) (1.1\%), and metaplastic types (0.9\%). At SGPGIMS Lucknow too, IDC is the commonest variety, with very few non-invasive (DCIS) cancers. The average breast tumor size in most centers is well over $5 \mathrm{~cm}$, with almost $2 / 3$ of these being grade III or II. At a major cancer center in the city of Mumbai, $70 \%$ patients were reported as having grade III disease [25]. A high proportion of patients were reported to have a high S-phase fraction, aneuploidy and other bad prognostic features on histology. At SGPGI Lucknow, the average tumor size has been $5.4 \mathrm{~cm}$. The Indian cancer registries too provide similar figures. The presentation seems similar in many other developing countries [26].

Estrogen (ER) and progesterone receptors (PR) are found positive in only $20-45 \%$ of Indian patients. ER-positive rates were reported to be lower in Indian patients than those in western countries. Not all patients in India undergo hormonal receptor testing as evident from the study in Delhi which showed only $35.5 \%$ of patients had receptor testing [27]. At
SGPGIMS, over the last decade, only about $45 \%$ patients have been found ER- and/or PR-positive (ER+/PR+). At TMH Mumbai, the ER+ status was found in $33 \%$, and PR+ in $46 \%$ of patients [28]. According to some, the low ER+ and PR+ status in Indian patients may actually be due to improper immunostaining techniques used. A study from a major hospital in Mumbai reported that the ER-/PR+ reported on IHC were actually due to suboptimal manual assays, and when the same tumors were evaluated using well standardized international kits, they were found ER+/PR+ [29]. HER2/neu or c-erb-B2 status - another clinically useful variable - is found positive in about $20 \%$ of Indian patients (SGPGIMS Lucknow data).

\section{Breast Cancer Screening in India}

No national or regional breast cancer screening program exists in India. At present, a dedicated breast cancer screening by clinical breast examination or mammography is not available outside research studies at a few institutions, or to women self-presenting to specialist hospitals to have these services provided for a fee. Mammography is available in a large number of public and private hospitals in almost all towns as a diagnostic service, which also provides a means for opportunistic screening for women willing to pay for it. Mammography is not advocated for mass screening, and it is generally felt that it may not be cost-effective in India [30]. Under the various public health initiatives, such as 'Health for All' and the National Rural Health Mission, emphasis is put on breast awareness and breast self-examination as a first step towards creating the ground work for a nationwide breast cancer screening program. It is felt that breast self-examination and clinical examination are perhaps the right tools for screening the huge population of India, but no credible data is available today to base these views on. Breast awareness and virtues of periodic breast self-examination are being promoted for early detection of breast cancer through print, electronic media, as well as through health personnel in various settings.

\section{Breast Cancer Awareness Programs}

TMH Mumbai has been involved in a randomized controlled trial $(n=150,000)$ which compares the efficacy of health education and clinical breast examination provided by trained primary health care workers with that of screening mammography. This study has now entered its 6th year and 3rd round of screening. The study already shows a good compliance-toscreening rate $(70 \%)$, and downstaging is evident. There are numerous other initiatives making a small difference in their own ways. In the city of Mumbai, an innovative way of promoting breast cancer awareness is available by way of a doorto-door mobile service to those who have registered with area coordinators for the non-governmental organization (NGO) 
'Helping Hand 4 Cancer Care'. The check-up and consultation together cost approximately 100 rupees (US\$2.5) a year. Numerous non-governmental and charity organizations lend a helping hand to breast cancer patients in coping with the suffering of treatment and lifestyle issues after treatment. The SGPGI Breast Health Initiative is one such organization involved with screening, early detection, patient and doctors' education, and also in collaborative multicenter research to attempt deriving 'socially and culturally acceptable and cost-effective solutions for the local problems'. Through its website www.sgpgibreasthealth.org - the organization provides an email and telephone helpline for patients to get a free second opinion, as well as for healthy women to seek advice about various breast health issues including breast cancer screening. The Prashanti Cancer Care Mission Pune, and the Cancer Patients Aid Association (CPAA) Mumbai are other such organizations. Manipal Hospital Bangalore runs a breast cancer awareness and screening program. Another helpline for breast cancer is run by the Indian Society of Medical and Paediatric Oncology (ISMPO) in Pune, which provides information and support to breast cancer patients.

In the coming years, the most important steps needed are creating awareness about breast cancer risk factors, early detection, along with making screening available to the population at risk, and providing multimodality treatment available to the majority at an affordable cost. The needs of the coming decades would perhaps be better served by small community cancer centers which are cost-effective and can manage most cancer patients in their own localities.

\section{Breast Cancer Treatment in India}

Due to a large variation in the health care standards between regions, the quality of treatment available for breast cancer patients varies from pathetic to world-class. In an acknowledgement of this fact, a recent effort has been made to chalk out national breast cancer management guidelines, taking into account the available data on breast cancer patients, and also keeping the logistics and economics in mind. The ICMR/WHO national breast cancer management protocols are still at a primitive stage, and far from being operationalized on a country-wide basis. The quality of breast cancer treatment is dictated by many factors, besides a patient's own outlook, and may include where the patient lives, which institution can she reach, what can she afford to spend on her treatment, whom does she trust, etc. Few patients are treated at well-equipped centers in a protocol-based manner, with numerous compromises made in the multimodality therapy, based on factors such as the economics, tolerance, nutritional deprivation, etc. For a huge number of patients, the number of treatment facilities is low. The poor compliance to treatment and follow-up is due to the social stigma attached to the disease, and the high cost of treatment [2]. Though there is no shortage of skills and expertise in cytology, the use of fine needle aspiration cytology is under-utilized. Pre-operative diagnosis is still based predominantly on clinical and incisional or excisional biopsy.

\section{Breast Cancer Surgery in India}

A large proportion of Indian patients are treated with inadequate/inappropriate initial surgical procedures before they are seen and managed by specialists. In a study from a major North Indian teaching hospital, almost $75 \%$ of the patients referred for management of operable early breast cancers (EBC) had had an incisional or excisional biopsy not intended for treatment of breast cancer [31]. At SGPGIMS Lucknow, the picture is more or less the same, and about $40 \%$ patients with EBC or locally advanced breast cancers present after some sort of surgical procedure which was either not intended to be a breast cancer treatment procedure or was inadequate. The quality of pathology reporting on breast lumpectomy/biopsy specimens and even on mastectomy and axillary clearance specimens leaves a lot to be desired. The lack of information on histological details, margins, number of axillary lymph nodes sampled, etc. results in considerable difficulty in interpretation of the extent of surgical treatment already performed. The inadequate and poor quality initial surgery and incomplete pathology reporting leads to a large proportion of patients managed at referral centers having stage Tx tumors. Such patients referred following an inadequate operation often pose a challenge in terms of the evaluation of the need/extent of re-operation in form of a revision wide local excision, axillary clearance alone, a repeat modified radical mastectomy (MRM), or even for planning adjuvant treatment $[2,31]$. Though the data on such patients are inconsistent, some reports suggest that such patients may be associated with worse outcomes as compared to the patients managed in an appropriate protocol-based manner in the first place [31]. Mastectomy is the predominant procedure for almost all stages of breast cancers [32]. MRM after or without neoadjuvant chemotherapy (NACT) is the norm in most centers. The completeness of mastectomy and axillary clearance is questionable. At TMH Mumbai, almost $40 \%$ of patients referred for management following a so called MRM in the hands of general surgeons at centers of various levels of expertise have to undergo a re-operation for completion mastectomy and/or axillary clearance. Very few patients are offered post-mastectomy reconstruction, and a small proportion of those offered accept to have it performed, with the reconstructive procedure being seen as an unnecessary burden of the scarce financial resources, and undue prolongation of the treatment.

\section{Breast Conservation Surgery for Breast Cancer in India}

Breast conservation surgery (BCS) is currently estimated to be offered to a miniscule proportion of Indian breast cancer 
patients at a few select centers. It is estimated that of the 100,000 patients that are treated for breast cancer every year in India, only about 1,000 are treated with breast-conserving treatment. The overall low rate of BCS in Indian patients is a reflection of the relatively late stage at presentation, and few centers are equipped to provide high-quality BCS with all its components including radiation therapy and pathology. Also, there is low acceptance on the part of the patients [25, 27]. A study from Delhi [27] showed only $11.3 \%$ underwent BCS, while MRM was performed in $88.7 \%$ of patients. Access to a radiotherapy facility was shown to influence the use of BCS [27]. At SGPGIMS Lucknow, almost $60 \%$ of EBC patients currently are treated with BCS. The overall low rates of breast conservation are due to the late presentation and low acceptance [27]. The Breast Unit at TMH Mumbai reports that the proportion of cases undergoing BCS has shown a significant upward trend from $12.6 \%$ in 1997 to $59.3 \%$ in 2001 [25]. Similar trends have been reported at SGPGIMS Lucknow, with more than $2 / 3$ of EBC patients accepting BCS in the recent 5 years, compared to only $15 \%$ in the years $1997-2002$. With encouraging results in EBC, BCS is also being offered to patients with locally advanced breast cancer after downstaging with NACT. Innovations to make BCS practical and safer in large locally advanced breast cancers have been reported [4]. Newer surgical and diagnostic procedures are available only at a few select high-quality centers. Sentinel lymph node biopsy using a dual color dye and radio-pharmaceutical technique is known to be performed at 7 centers only. There may be many more where the sentinel lymph node biopsy using blue dye alone may be performed. It is estimated that only about 500 patients per year undergo this procedure.

\section{Systemic and Adjuvant Treatment of Breast Cancer in India}

A wide variety of chemotherapy regimens are in use. While most agree that anthracycline-based combination chemotherapy is the appropriate first line chemotherapy for most patients, based on acknowledgement of the fact that anthracycline-based regimens (5-fluorouracil (5-FU), doxorubicin, cyclophosphamide - FAC) result in survival benefit when compared to the cyclophosphamide-methotrexate-5FU combination $(\mathrm{CMF})$ with improvement in annual odds of recurrence and death by 11 and $12 \%$, respectively. The FAC regimen is substantially costlier than $\mathrm{CMF}$, and the cost difference is even more contrasting in view of the need for management of anthracycline toxicity. As a result, $\mathrm{CMF}$ is still preferred for a large number of unaffording and elderly patients. The choice of systemic chemotherapy and hormone treatment is dictated by the paying capacity of the patient, as the majority have to buy the medicines by themselves in absence of governmental or insurance support. However, in recent times, the support towards chemotherapy from the federal and state govern- ments as well as from numerous charitable organizations has been increasingly available for needy patients. A study [32] conducted in a community hospital in Southern India on 122 patients reported IDC in $96.7 \%$ of patients, $27 \%$ being in the premenopausal age group, EBC as well as metastatic breast cancer in $6.8 \%$ each; mastectomy was done in $93.8 \%$ and BCS in $6.3 \%$ of patients. Chemotherapy regimens used were FAC in $51.2 \%$ and $\mathrm{CMF}$ in $48.8 \%$ of patients. Use of taxanes is limited by their high costs and higher toxicity. Tamoxifen is the most widely used endocrine therapy for breast cancer in India, and the proportion of post-menopausal patients treated with aromatase inhibitors, though increasing, is very small. Surgical oopherectomy still widely practiced as a rapid and highly effective ablative endocrine treatment, and is a valid choice in the vast majority of pre-menopausal and advanced stage breast cancer patients. Radiation ablation and medical ablative treatment such as goserlin are seen as expensive and relatively less effective. Targeted biological therapies like trastuzumab are out of reach for most Indian patients. There are very few dedicated radiation therapy units, and as a result, it is estimated that only about $20 \%$ of patients are offered post-mastectomy radiation therapy. The paucity of radiation therapy set-ups is a deterrent in setting up more breast conservation therapy programs. Compliance with adjuvant treatment amongst Indian patients outside major institutions is rather poor. This is because of the high cost, long time duration, high toxicity, and the impaired wellbeing systemic cytotoxic chemotherapy and radiation therapy result in.

\section{Survival Estimates for Indian Breast Cancer Patients}

The 5-year overall survival rate in the Bangalore PBCR has been reported at $42.3 \%$ [6]. The Madras Metropolitan Tumor Registry reported survival rates of 80,58 , and $48 \%$ at 1,3 , and 5 years, respectively [33]. A study from Kerala showed a 5-year survival rate of 40\% [22]. Chopra [3] from New Delhi reported a 5-year overall survival rate of $55 \%$, with $80 \%$ EBC, 45-60\% locally advanced, and 20\% MBC patients estimated to be alive at 5 years [3]. At SGPGIMS Lucknow, the 5-year overall survival rate has been $62 \%$. The 5 -year actuarial survival has been $90 \%$ for stage I patients, $78 \%$ for stage II, $57 \%$ for stage III, and $22 \%$ for stage IV, though the survival data is available based on the follow-up information for $75 \%$ patients only. The overall 10-year estimated survival of SGPGIMS Lucknow patients was $35 \%$, with $75 \%$ of stage I, $55 \%$ of stage II, $35 \%$ of stage III, and $5 \%$ of stage IV patients estimated to be alive at 10 years.

\section{Summary}

The incidence of breast cancer in Indian women is much lesser than the counterparts in industrialized nations. Nonetheless, 
breast cancer has emerged as the commonest cancer in urban Indian women, and second commonest to the cancer of the uterine cervix in rural women. Like the overall poor health awareness, awareness of breast cancer is poor. This, along with a general indifference towards women's health, the lack of breast cancer screening programs, and delay in seeking advice for a recognized health problem due to financial and social reasons, result in delayed diagnosis. As a result, Indian breast cancer patients present with advanced disease stage and have numerous poor prognostic factors such as large tumor, lymph node metastases, high pathological grade and poor hormone receptors status. Besides, the poor access to high-quality multimodality treatment facilities, few treatment facilities for a huge population, and poor financial resources on the patient's part as well as the health care provider's part result in compromised quality of care to patients with established disease. It is thus not surprising that Indian breast cancer patients have higher locoregional recurrences and poorer overall survival. The recent emphasis by governmental agencies, institutions, and non-governmental and charity organizations on improved health awareness, promotion of early detection, providing comprehensive multimodality treatment in a protocol-based manner, and providing support for breast cancer management as well as for screening and rehabilitation have resulted in an improving trend with more and more early stage cancers being diagnosed and treated timely, resulting in improving survival and quality of life of Indian breast cancer patients.

\section{References}

1 National Cancer Registry Program: Ten year consolidated report of the Hospital Based Cancer Registries, 1984-1993, an assessment of the burden and care of cancer patients. Indian Council of Medical Research, New Delhi, 2001

$\checkmark 2$ Agarwal G, Pradeep PV, Aggarwal V, Yip CH, Cheung PS: Spectrum of breast cancer in Asian women. World J Surg 2007;31:1031-40.

3 Chopra R: The Indian scene. J Clin Oncol 2001;19 (18 suppl):106S-111S.

4 Aggarwal V, Agarwal G, Lal P, Krishnani N, Mishra A, Verma AK, Mishra SK: Feasibility study of safe breast conservation in large and locally advanced cancers with use of radiopaque markers to mark pre-neoadjuvant chemotherapy tumor margins. World J Surg 2007, Nov 21; Epub ahead of print.

5 Parkin DM: Global cancer statistics in the year 2000. Lancet Oncol 2001;2:533.

$\checkmark 6$ Nandakumar A, Anantha N, Venugopal TC, Sankaranarayanan R, Thimmasetty K, Dhar M: Survival in breast cancer: a population-based study in Bangalore, India. Int J Cancer 1995;60:593-6.

7 National Cancer Registry Programme: Consolidated report of the population based cancer registries 1990-1996. Indian Council of Medical Research, New Delhi, 2001.

8 Saxena S, Szabo CI, Chopin S, Barjhoux L, Sinilnikova O, Lenoir G, Goldgar DE, Bhatanager: BRCA1 and BRCA2 in Indian breast cancer patients. Hum Mutat 2002;20:473-4.

9 Murthy NS, Agarwal UK, Chaudhry K, Saxena S: A study on time trends in incidence of breast cancer Indian scenario. Eur J Cancer Care 2007;16:185-6.

10 Dinshaw KA, Sarin R, Budrukkar AN, Shrivastava SK, Deshpande DD, Chinoy RF, Badwe R, Hawaldar R: Safety and feasibility of breast conserving therapy in Indian women: two decades of experience at Tata Memorial Hospital. J Surg Oncol 2006;94:105-13.

11 Mathew A, Pandey M, Rajan B: Do younger women with non-metastatic and non-inflammatory breast carcinoma have poor prognosis? World J Surg Oncol 2004;2:2.

12 Shavers VL, Harlan LC, Stevens JL: Racial/ethnic variation in clinical presentation, treatment, and survival among breast cancer patients < age 35 . Cancer 2003;97:134-47.
Paymaster JC, Gangadharan P: Some observations on the epidemiology of cancer of breast in women in western India. Int J Cancer 1972;10:443-50.

14 Rao DN, Ganesh B, Desai PB: Role of reproductive factors in breast cancer in a low-risk area: a case-control study. Br J Cancer 1994;70:129-32.

15 Saxena S, Rekhi B, Bansal A, Bagga A, Chintamani , Murthy NS: Clinico-morphological patterns of breast cancer including family history in a New Delhi hospital, India - a cross-sectional study. World J Surg Oncol 2005;3:67.

16 Valarmathi MT, A A, Deo SS, Shukla NK, Das SN: BRCA1 germline mutations in Indian familial breast cancer. Hum Mutat 2003;21:98-9.

17 Kumar BV, Lakhotia S, Ankathil R, Madhavan J, Jayaprakash PG, Nair MK, Somasundaram K: Germline BRCA1 mutation analysis in Indian breast/ovarian cancer families. Cancer Biol Ther 2002;1:18-21.

18 Rajkumar T, Soumittra N, Nancy NK, Swaminathan R, Sridevi V, Shanta V: BRCA1, BRCA2 and CHEK2 (1100 del C) germline mutations in hereditary breast and ovarian cancer families in South India. Asian Pac J Cancer Prev 2003;4:203-8.

19 Hedau S, Jain N, Husain SA, Mandal AK, Ray G, Shahid M, Kant R, Gupta V, Shukla, NK, Deo SS, Das BC: Novel germline mutations in breast cancer susceptibility genes BRCA1, BRCA2 and p53 gene in breast cancer patients from India. Breast Cancer Res Treat 2004;88:177-86.

20 Valarmathi MT, Sawhney M, Deo SS, Shukla NK, Das SN: Novel germline mutations in the BRCA1 and BRCA2 genes in Indian breast and breastovarian cancer families. Hum Mutat 2004;23:205.

21 Paymaster TC, Gangadharan P: Cancer in Parsi community of Bombay. Int J Cancer 1970;5:426-31.

22 Nair MK, Sankaranarayanan R, Nair KS, Amma NS, Varghese C, Padmakumari G, Cherian T: Overall survival from breast cancer in Kerala, India, in relation to menstrual, reproductive, and clinical factors. Cancer 1993;71:1791-6.

23 El Saghir NS, Khalil MK, Eid T, El Kinge AR, Charafeddine M, Geara F, Seoud M, Shamseddine AI: Trends in epidemiology and management of breast cancer in developing Arab countries: a literature and registry analysis. Int J Surg 2007;5:225-33.
24 Gajalakshmi CK, Shanta V, Hakama M: Risk factors for contralateral breast cancer in Chennai (Madras), India. Int J Epidemiol 1998;27:743-50.

25 Dinshaw KA, Budrukkar AN, Chinoy RF, Sarin R, Badwe R, Hawaldar R, Shrivastava SK: Profile of prognostic factors in 1022 Indian women with early-stage breast cancer treated with breast-conserving therapy. Int J Radiat Oncol Biol Phys 2005; 63:1132-41.

26 Aziz Z, Sana S, Akram M, Saeed A: Socioeconomic status and breast cancer survival in Pakistani women. J Pak Med Assoc 2004;54:448-53.

27 Raina V, Bhutani M, Bedi R, Sharma A, Deo SV, Shukla NK, Mohanti BK, Rath GK: Clinical features and prognostic factors of early breast cancer at a major cancer center in North India. Indian J Cancer 2005;42:40-5.

28 Desai SB, Moonim MT, Gill AK, Punia RS, Naresh KN, Chinoy RF: Hormone receptor status of breast cancer in India: a study of 798 tumors. Breast 2000; 9:267-70.

29 Navani S, Bhaduri AS: High incidence of oestrogen receptor negative progesterone receptor positive phenotype in Indian breast cancer: fact or fiction? Indian J Pathol Microbiol 2005;48:199-201.

30 Mittra I: Breast screening: the case for physical examination without mammography. Lancet 1994; 343:342-4.

31 Tewari M, Pradhan S, Kumar M, Shukla HS: Effect of prevailing local treatment options of breast cancer on survival outside controlled clinical trials: experience of a specialist breast unit in North India. World J Surg 2006;30:1794-801.

32 Kuraparthy S, Reddy KM, Yadagiri LA, Yutla M, Venkata PB, Kadainti SV, Reddy RP: Epidemiology and patterns of care for invasive breast carcinoma at a community hospital in Southern India. World J Surg Oncol 2007;5:56.

33 Gajalakshmi CK, Shanta V, Swaminathan R, Sankaranarayanan R, Black RJ: A population-based survival study on female breast cancer in Madras, India. Br J Cancer 1997;75:771-5. 